\title{
Incorporating Non-Convex Operating Characteristics into Bi-Level Optimization Electricity Market Models
}

Ye, Yujian; Papadaskalopoulos, Dimitrios; Kazempour, Jalal; Strbac, Goran

Published in:

IEEE Transactions on Power Systems

Link to article, DOI:

10.1109/TPWRS.2019.2925317

Publication date:

2019

Document Version

Peer reviewed version

Link back to DTU Orbit

Citation (APA):

Ye, Y., Papadaskalopoulos, D., Kazempour, J., \& Strbac, G. (2019). Incorporating Non-Convex Operating Characteristics into Bi-Level Optimization Electricity Market Models. IEEE Transactions on Power Systems, 35(1), 163 - 176. https://doi.org/10.1109/TPWRS.2019.2925317

\section{General rights}

Copyright and moral rights for the publications made accessible in the public portal are retained by the authors and/or other copyright owners and it is a condition of accessing publications that users recognise and abide by the legal requirements associated with these rights.

- Users may download and print one copy of any publication from the public portal for the purpose of private study or research.

- You may not further distribute the material or use it for any profit-making activity or commercial gain

- You may freely distribute the URL identifying the publication in the public portal 


\section{Incorporating Non-Convex Operating Characteristics into Bi-Level Optimization Electricity Market Models}

Yujian Ye, Member, IEEE, Dimitrios Papadaskalopoulos, Member, IEEE, Jalal Kazempour, Senior Member, IEEE, and Goran Strbac, Member, IEEE

\begin{abstract}
Bi-level optimization constitutes the most popular mathematical methodology for modeling the deregulated electricity market. However, state-of-the-art models neglect the physical non-convex operating characteristics of market participants, due to their inherent inability to capture binary decision variables in their representation of the market clearing process, rendering them problematic in modeling markets with complex bidding and unit commitment (UC) clearing mechanisms. This paper addresses this fundamental limitation by proposing a novel modeling approach enabling incorporation of these non-convexities into bi-level optimization market models, which is based on the relaxation and primal-dual reformulation of the original, nonconvex lower level problem and the penalization of the associated duality gap. Case studies demonstrate the ability of the proposed approach to closely approximate the market clearing solution of the actual UC clearing algorithm and devise more profitable bidding decisions for strategic producers than the state-of-theart bi-level optimization approach, and reveal the potential of strategic behavior in terms of misreporting non-convex operating characteristics.
\end{abstract}

Index Terms-Bi-level optimization, electricity markets, nonconvexities, strategic bidding, unit commitment.

\section{NOMENCLATURE}

\section{A. Indices and Sets}

$t \in T \quad$ Index and set of time periods

$i \in I \quad$ Index and set of producers

$i$ - Index of producers other than $i$

$j \in J \quad$ Index and set of demands

$b \in B \quad$ Index and set of generation blocks

$c \in C \quad$ Index and set of demand blocks

$V^{P} \quad$ Set of decision variables of the original lower level (LL) problem

$V^{D} \quad$ Set of decision variables of the dual of the relaxed LL problem

Set of decision variables of the final single-level optimization problem

Yujian Ye, Dimitrios Papadaskalopoulos, and Goran Strbac are with the Department of Electrical and Electronic Engineering, Imperial College London, London, U.K. (email: yujian.ye11@imperial.ac.uk, d.papadaskalopoulos08@imperial.ac.uk,g.strbac@imperial.ac.uk).

Jalal Kazempour is with the Technical University of Denmark, Kongens Lyngby 2800, Denmark (e-mail: seykaz@elektro.dtu.dk).

This work has been supported by the SysFlex project which has received funding from the European Union's Horizon 2020 research and innovation program.

\section{B. Parameters}

$N_{T} \quad$ Length of market horizon

$\lambda_{i, b}^{G} \quad$ Marginal cost of block $b$ of producer $i(\mathfrak{f} / \mathrm{MWh})$

$f_{i} \quad$ No-load cost of producer $i(\mathfrak{f} / \mathrm{h})$

$K_{i}^{U}, K_{i}^{D} \quad$ Start-up / shut-down cost of producer $i(\mathfrak{f} / \mathrm{h})$

$g_{i}^{\text {min }} \quad$ Minimum stable generation limit of producer $i$ (MW)

$g_{i, b}^{\max } \quad$ Maximum generation limit of block $b$ of producer $i$ (MW)

$R_{i}^{U}, R_{i}^{D} \quad$ Ramp-up / down limit of producer $i(\mathrm{MW} / \mathrm{h})$

$T_{i}^{U}, T_{i}^{D} \quad$ Minimum-up / down time limit of producer $i(\mathrm{~h})$

$g_{i, 0} \quad$ Initial output of producer $i$ (MW)

$u_{i, 0} \quad$ Initial UC status of producer $i$

$H_{i}^{U}, H_{i}^{D} \quad$ Number of periods producer $i$ must be initially on / off due to its minimum-up / down limits.

$k_{i}^{\max } \quad$ Upper limit of strategic bidding variable of producer $i$

$\lambda_{j, t, c}^{D} \quad$ Marginal benefit of block $c$ of demand $j$ at period $t$ (£/MWh)

$d_{j, t, c}^{\max } \quad$ Maximum demand limit of block $c$ of demand $j$ at period $t(\mathrm{MW})$

C. Variables

$g_{i, t, b} \quad$ Power output of block $b$ of producer $i$ at period $t$ (MW)

$u_{i, t} \quad$ Binary UC status of producer $i$ at period $t\left(u_{i, t}=\right.$ 1 if it is on, $u_{i, t}=0$ if it is off)

$C_{i, t}^{U}, C_{i, t}^{D} \quad$ Start-up / shut-down cost incurred by producer $i$ at period $t(£ / h)$

$k_{i} \quad$ Strategic bidding variable of producer $i$

$d_{j, t, c} \quad$ Power input of block $c$ of demand $j$ at period $t$ (MW)

$\lambda_{t} \quad$ Market clearing price at period $t(£ / \mathrm{MWh})$

\section{INTRODUCTION}

\section{A. Background and Motivation}

S IGNIFICANT efforts towards the deregulation of the electricity industry have been witnessed worldwide during the last decades, characterized by the unbundling of vertically integrated utilities and the introduction of competition among 
multiple self-interested market participants [1]. This paradigm change means that traditional centralized models, optimizing system objectives (e.g. minimizing system costs or maximizing social welfare) and assuming perfectly competitive (pricetaking) behavior by market participants, are not able to provide accurate and meaningful insights anymore. New models are required instead, capable of capturing the strategic, profitdriven behavior of self-interested market players and identifying the market outcomes emerging from the interactions of these players.

Bi-level optimization has clearly been the most successful methodological framework for developing such new models over the last two decades. The popularity of this methodology lies in its ability to capture in a mathematically rigorous fashion the interaction between the strategic decision making of self-interested players (modeled in the upper level) and the competitive clearing of the electricity market (modeled in the lower level). Bi-level optimization problems are usually solved after converting them to single-level Mathematical Programs with Equilibrium Constraints (MPEC), through the replacement of the lower level problem by its equivalent Karush-Kuhn-Tucker (KKT) optimality conditions.

This popularity is justified by the large number of papers employing this methodology, including papers [2]-[25]. This list includes papers focusing on market models including only traditional generation participants [2]-[16] as well as models investigating the impact of demand side flexibility [17]-[20] and energy storage [21]-[25] in electricity markets. Furthermore, some of these papers focus on the optimization of the strategic bidding decisions of a single market player [5], [8], [9], [12], [15], [16], [18], [19], [21]-[25], while others have gone further, aiming at identifying the market equilibria stemming from the interactions of multiple players [2]-[4], [6], [7], [10], [11], [13], [14], [17], [20]. Finally, some of these papers have managed to incorporate realistic aspects such as network constraints [2]-[6], [9], [11], [12], [14]-[18], [20]-[25], and uncertainty faced by market players [5], [8][10], [12]-[16], [18], [19], [24], [25] in the developed market models.

However, this modeling framework exhibits a fundamental limitation which is yet to be comprehensively addressed. This is that the lower level problem does not include any binary decision variables since the derivation of the equivalent KKT optimality conditions is only possible when this problem is continuous and convex [26]. As a result, the models developed in all previous relevant papers [2]-[25] consider only the variable costs, maximum output limits and ramp rates of generation units and they neglect physical non-convex cost components and constraints that are associated with binary commitment decisions, including no-load, start-up and shutdown costs, minimum stable generation limits, and minimumup / down time constraints.

However, these complex operating characteristics affect the market clearing outcome determined by the lower level problem and consequently the strategic decisions of the market players determined by the upper level problem. This implies that the employment of state-of-the-art bi-level optimization market models may lead to sub-optimal bidding decisions for strategic players as well as calculation of inaccurate market equilibria when the interaction of multiple players is modeled. This limitation is particularly important when modeling markets with complex bidding mechanisms, whose clearing algorithm involves the solution of a mixed-integer unit commitment (UC) problem, such as many markets in the USA (e.g. California, PJM, New York, MISO) [27]-[30], and Europe (e.g. Greece, Poland, Ireland \& Northern Ireland) [31].

Two recent pieces of work have proposed mathematical approaches to solve bi-level optimization problems with binary variables in the lower level problem, although they have applied these approaches to fundamentally different problems than the strategic bidding problem examined in papers [2][25] and this paper. The first one involves an iterative solution technique based on a column-and-constraint generation method [32], [33]. Despite its relevance, this approach has been applied to a class of bi-level optimization problems with a different mathematical structure that the one of papers [2][25]. When applied to a strategic bidding bi-level optimization problem, the market clearing prices become unbounded decision variables and independent of the strategic players' bidding decisions. This contradicts the very essence of the application of bi-level optimization to electricity market modeling problems and renders the obtained results unreliable. The second one derives KKT optimality conditions for each combination of the (fixed) states of all the binary variables in the problem [34]. Despite the theoretical optimality of the obtained solution, this approach is practically inapplicable to realistic instances of the strategic bidding problem due to its enormous computational complexity, since the number of possible combinations and therefore the number of KKT optimality conditions increase exponentially with the number of players and the number of time periods in the market horizon.

\section{B. Scope and Contributions}

This paper aims at addressing this fundamental limitation by proposing a novel modeling approach that enables incorporation of the non-convex generation operating characteristics, associated with binary UC decisions, into the market clearing representation of bi-level optimization market models. This approach is based on the relaxation and primal-dual reformulation of the original, non-convex lower level problem and the penalization of the associated duality gap. Although this approach can be potentially applied to all types of market problems investigated in the relevant literature [2]-[25], the authors have chosen for clarity reasons to apply it to the simplest instance of these market problems, i.e. the optimization of the strategic bidding decisions of a single producer in the electricity market, neglecting aspects such as network constraints, demand flexibility, energy storage participation and uncertainty effects.

Case studies on a test market with day-ahead horizon and hourly resolution demonstrate the effectiveness and value of the proposed approach by carrying out three different types of tests. The first one demonstrates the ability of the proposed model to closely approximate the market clearing solution of the actual UC clearing algorithm. The second one 
demonstrates the value of the proposed model in devising more profitable bidding decisions for strategic producers participating in a complex bidding market than state-of-the-art bi-level optimization models. The third one demonstrates the ability of the proposed model to reveal and quantitatively analyze the potential of strategic behavior in terms of misreporting nonconvex operating characteristics, which cannot be explored with state-of-the-art models.

\section{Paper Structure}

The rest of this paper is organized as follows. Section II details the proposed modeling approach. Section III presents case studies demonstrating the value of the proposed approach. Finally, Section IV discusses conclusions and future extensions of this work.

\section{Proposed Modeling Approach}

\section{A. Modeling Assumptions}

For clarity reasons, the main assumptions behind the proposed model are outlined below:

1) The modeled market is a pool-based, energy-only market with a complex bidding mechanism, implying that the producers submit price-quantity bids and their UC constraints. The clearing algorithm involves the solution of a mixed-integer UC problem, maximizing the perceived social welfare, similar to the ones employed in [27]-[30]. The pricing mechanism is the one proposed in [35], where the clearing prices are obtained by solving a continuous version of the clearing algorithm, with the binary UC variables fixed to their optimal values. Finally, side payments (uplift payments) are not explicitly considered in the examined modeling framework.

2) For presentation clarity reasons and without loss of generality, we assume that each producer $i$ owns a single generation unit.

3) As discussed in Section I-B, the examined market problem lies in the optimization of the strategic bidding decisions of a single producer $i$. Following the model employed in [3], [4], [6], [17], [20] the strategic behavior of producer $i$ is expressed through a decision variable $1 \leq k_{i} \leq k_{i}^{\max }$. If $k_{i}=1$, producer $i$ behaves competitively and offers its actual marginal costs $\lambda_{i, b}^{G}, \forall b$ to the market. If $1<k_{i} \leq k_{i}^{\max }$, producer $i$ behaves strategically and offers higher than its actual marginal costs $\left(k_{i} * \lambda_{i, b}^{G}, \forall b\right)$ to the market. Producer $i$ should optimize the value of $k_{i}$ by accounting for the tradeoff between higher market clearing price and lower clearing quantity. More specifically, a higher $k_{i}$ will tend to increase market prices, but at the same time it will tend to decrease the quantity sold by producer $i$, since producers with lower submitted offers may replace $i$ in the merit order and / or the demand side may reduce demand.

4) Each demand submits to the market a non-increasing (capturing the effect of demand's self-price elasticity) stepwise bid curve, consisting of a number of blocks.

\section{B. Bi-level Optimization Model Considering Non-convex Op- erating Characteristics}

Following the paradigm of the relevant literature [2]-[25], our starting point for addressing the market problem discussed in point 3) above, is a bi-level optimization model. However, as discussed in Section I-A, state-of-the-art models neglect physical non-convex operating characteristics of the producers. Therefore, in this section, we present a new bi-level optimization model considering these non-convexities. This model, optimizing the strategic bidding decisions of producer $i$, is formulated as follows:

(Upper level)

$$
\begin{gathered}
\max _{\left\{k_{i}\right\}} \sum_{t, b} \lambda_{t} g_{i, t, b}-\sum_{t, b} \lambda_{i, b}^{G} g_{i, t, b} \\
-\sum_{t} f_{i} u_{i, t}-\sum_{t} C_{i, t}^{U}-\sum_{t} C_{i, t}^{D}
\end{gathered}
$$

subject to:

$$
1 \leq k_{i} \leq k_{i}^{\max }
$$

(Lower level)

$$
\begin{gathered}
\min _{V^{P}}\left[\left(\sum_{t, b} k_{i} \lambda_{i, b}^{G} g_{i, t, b}+\sum_{t} f_{i} u_{i, t}+\sum_{t} C_{i, t}^{U}\right.\right. \\
\left.+\sum_{t} C_{i, t}^{D}\right)+\left(\sum_{i-, t, b} \lambda_{i-, b}^{G} g_{i-, t, b}+\sum_{i-, t} f_{i-} u_{i-, t}\right. \\
\left.\left.+\sum_{i-, t} C_{i-, t}^{U}+\sum_{i-, t} C_{i-, t}^{D}\right)-\sum_{j, t, c} \lambda_{j, t, c}^{D} d_{j, t, c}\right]
\end{gathered}
$$

where:

$$
V^{P}=\left\{g_{i, t, b}, u_{i, t}, C_{i, t}^{U}, C_{i, t}^{D}, d_{j, t, c}\right\}
$$

subject to:

$$
\begin{aligned}
& \sum_{j, c} d_{j, t, c}-\sum_{i, b} g_{i, t, b}=0: \lambda_{t}, \forall t \\
& 0 \leq g_{i, t, b} \leq u_{i, t} g_{i, b}^{\max }: \mu_{i, t, b}^{\min }, \mu_{i, t, b}^{\max }, \forall i, \forall t, \forall b \\
& 0 \leq d_{j, t, c} \leq d_{j, t, c}^{\max }: \nu_{j, t, c}^{\min }, \nu_{j, t, c}^{\max }, \forall j, \forall t, \forall c \\
& u_{i, t} g_{i}^{\text {min }} \leq \sum_{b} g_{i, t, b}: \xi_{i, t}^{\min }, \forall i, \forall t \\
& \sum_{b} g_{i, t, b}-\sum_{b} g_{i,(t-1), b} \leq R_{i}^{U}: \pi_{i, t}^{U}, \forall i, \forall t \\
& \sum_{b} g_{i,(t-1), b}-\sum_{b} g_{i, t, b} \leq R_{i}^{D}: \pi_{i, t}^{D}, \forall i, \forall t \\
& C_{i, t}^{U} \geq 0: \rho_{i, t}^{U}, \forall i, \forall t \\
& C_{i, t}^{D} \geq 0: \rho_{i, t}^{D}, \forall i, \forall t \\
& C_{i, t}^{U} \geq\left(u_{i, t}-u_{i,(t-1)}\right) K_{i}^{U}: \sigma_{i, t}^{U}, \forall i, \forall t \\
& C_{i, t}^{D} \geq\left(u_{i,(t-1)}-u_{i, t}\right) K_{i}^{D}: \sigma_{i, t}^{D}, \forall i, \forall t \\
& \sum_{t=1}^{H_{i}^{U}}\left(1-u_{i, t}\right)=0: \tau_{i}^{U}, \forall i \\
& \sum_{t=1}^{H_{i}^{D}} u_{i, t}=0: \tau_{i}^{D}, \forall i \\
& T_{i}^{U}\left(u_{i, t}-u_{i,(t-1)}\right) \leq \sum_{r=t}^{t+T_{i}^{U}-1} u_{i, r}: \phi_{i, t}^{U}, \\
& \forall i, \forall t=H_{i}^{U}+1, \ldots, N_{T}-T_{i}^{U}+1
\end{aligned}
$$




$$
\begin{gathered}
-T_{i}^{D}\left(u_{i, t}-u_{i,(t-1)}\right) \leq \sum_{r=t}^{t+T_{i}^{D}-1}\left(1-u_{i, r}\right): \phi_{i, t}^{D}, \\
\forall i, \forall t=H_{i}^{D}+1, \ldots, N_{T}-T_{i}^{D}+1 \\
\sum_{r=t}^{N_{T}}\left(u_{i, r}-\left(u_{i, t}-u_{i,(t-1)}\right)\right) \geq 0: \chi_{i, t}^{U}, \\
\forall i, \forall t=N_{T}-T_{i}^{U}+2, \ldots, N_{T} \\
\sum_{r=t}^{N_{T}}\left(1-u_{i, r}-\left(u_{i,(t-1)}-u_{i, t}\right)\right) \geq 0: \chi_{i, t}^{D}, \\
\forall i, \forall t=N_{T}-T_{i}^{D}+2, \ldots, N_{T} \\
u_{i, t}=\{0,1\}, \forall i, \forall t .
\end{gathered}
$$

The upper level (UL) problem determines the optimal bidding strategy of producer $i$ so as to maximize its profit (1a). This problem is subject to the limits of the strategic bidding variable (1b) and the lower level (LL) problem (1c)(1u). The latter represents the market clearing algorithm, minimizing the perceived negative social welfare (1c), subject to demand-supply balance constraints (1e), producers' and demands' power bounds (1f)-(1g), minimum stable generation constraints (1h), ramp-up and ramp-down constraints (1i)(1j), start-up and shut-down cost constraints (1k)-(1n), and minimum-up and minimum-down time constraints (1o)-(1t).

In contrast with state-of-the-art bi-level optimization models, this model includes the binary decision variables (1u) associated with the producers' UC status. Therefore, it is able to account for non-convex cost components (namely noload, start-up and shut-down costs) and non-convex operating constraints (namely minimum stable generation, minimum-up time and minimum-down time constraints).

\section{Reformulation of LL problem}

As discussed in Section I-A, state-of-the-art bi-level optimization problems are solved after converting them to singlelevel MPEC, through the replacement of the LL problem by its equivalent KKT optimality conditions [2]-[25]. This reformulation is possible since the LL problem in state-ofthe-art models is convex, as it neglects binary UC decisions and the associated non-convex operating characteristics of the producers. On the other hand, it is not applicable to the new bi-level optimization model (1) since the latter includes these binary decisions which prevent the derivation of equivalent KKT conditions.

In order to address this fundamental challenge, an alternative approach for the reformulation of the LL problem is adopted, based on [36]. The first step of this approach lies in the relaxation of the original LL problem (1c)-(1u) by relaxing its binary constraints $(1 \mathrm{u})$ as continuous constraints:

$$
0 \leq u_{i, t} \leq 1: \psi_{i, t}^{\min }, \psi_{i, t}^{\max }, \forall i, \forall t
$$

with $\psi_{i, t}^{\min }, \psi_{i, t}^{\max }$ being their respective dual variables. This relaxation enables the definition of dual variables for all the constraints of the LL problem, which are indicated after a colon in constraints (1e)-(1t) above. In other words, this relaxation coverts the LL problem from a mixed-integer linear problem to a continuous linear problem, defined by (1c)-(1t) and (2).

After this conversion, the dual problem associated with the relaxed LL problem can be derived, which is formulated as follows:

$$
\begin{gathered}
\max _{V^{D}}\left[-\sum_{j, t, c} \nu_{j, t, c}^{\max } d_{j, t, c}^{\max }-\sum_{i, t} \pi_{i, t}^{U} R_{i}^{U}-\sum_{i,(t=1)} \pi_{i, t}^{U} g_{i, 0}\right. \\
+\sum_{i,(t=1)} \pi_{i, t}^{D} g_{i, 0}-\sum_{i, t} \pi_{i, t}^{D} R_{i}^{D}-\sum_{i,(t=1)} \sigma_{i, t}^{U} K_{i}^{U} u_{i, 0} \\
+\sum_{i,(t=1)} \sigma_{i, t}^{D} K_{i}^{D} u_{i, 0}+\sum_{i} \tau_{i}^{U} H_{i}^{U} \\
-\left.\sum_{i,\left(t=H_{i}^{U}+1\right)} \phi_{i, t}^{U} T_{i}^{U} u_{i, 0}\right|_{H_{i}^{U}=0} \\
+\left.\sum_{i,\left(t=H_{i}^{D}+1\right)} \phi_{i, t}^{D} T_{i}^{D} u_{i, 0}\right|_{H_{i}^{D}=0}-\sum_{i, t=H_{i}^{D}+1}^{N_{T}-T_{i}^{D}+1} \phi_{i, t}^{D} T_{i}^{D} \\
\left.-\sum_{i, t=N_{T}-T_{i}^{D}+2}^{N_{T}} \chi_{i, t}^{D}\left(N_{T}-t+1\right)-\sum_{i, t} \psi_{i, t}^{\max }\right]
\end{gathered}
$$

where:

$$
\begin{gathered}
V^{D}=\left\{\lambda_{t}, \mu_{i, t, b}^{\max }, \nu_{j, t, c}^{\max }, \xi_{i, t}^{\min }, \pi_{i, t}^{U}, \pi_{i, t}^{D}, \sigma_{i, t}^{U},\right. \\
\left.\sigma_{i, t}^{D}, \tau_{i}^{U}, \tau_{i}^{D}, \phi_{i, t}^{U}, \phi_{i, t}^{D}, \chi_{i, t}^{U}, \chi_{i, t}^{D}, \psi_{i, t}^{\max }\right\}
\end{gathered}
$$

subject to:

$$
\begin{gathered}
k_{i} \lambda_{i, b}^{G}-\lambda_{t}+\mu_{i, t, b}^{\max }-\xi_{i, t}^{\min }+\pi_{i, t}^{U}-\pi_{i,(t+1)}^{U} \\
-\pi_{i, t}^{D}+\pi_{i,(t+1)}^{D} \geq 0, \forall i, \forall t<N_{T}, \forall b \\
k_{i} \lambda_{i, b}^{G}-\lambda_{t}+\mu_{i, t, b}^{\max }-\xi_{i, t}^{\min }+\pi_{i, t}^{U}-\pi_{i, t}^{D} \geq 0, \\
\forall i, \forall t=N_{T}, \forall b \\
\lambda_{i-, b}^{G}-\lambda_{t}+\mu_{i-, t, b}^{\max }-\xi_{i-, t}^{\min }+\pi_{i-, t}^{U}-\pi_{i-,(t+1)}^{U} \\
-\pi_{i-, t}^{D}+\pi_{i-,(t+1)}^{D} \geq 0, \forall i-, \forall t<N_{T}, \forall b \\
\lambda_{i-, b}^{G}-\lambda_{t}+\mu_{i-, t, b}^{\max }-\xi_{i-, t}^{\min }+\pi_{i-, t}^{U}-\pi_{i-, t}^{D} \geq 0, \\
\forall i-, \forall t=N_{T}, \forall b \\
f_{i}-\sum_{b} \mu_{i, t, b}^{\max } g_{i, b}^{\max }+\xi_{i, t}^{\min } g_{i}^{\min } \\
+\left(\sigma_{i, t}^{U}-\sigma_{i,(t+1)}^{U}\right) K_{i}^{U}-\left(\sigma_{i, t}^{D}-\sigma_{i,(t+1)}^{D}\right) K_{i}^{D} \\
+\psi_{i, t}^{\max }+\Gamma_{i, t}^{1} \geq 0, \forall i, \forall t<N_{T} \\
f_{i}-\sum_{b} \mu_{i, t, b}^{\max } g_{i, b}^{\max }+\xi_{i, t}^{\min } g_{i}^{\min } \\
+\sigma_{i, t}^{U} K_{i}^{U}-\sigma_{i, t}^{D} K_{i}^{D}+\psi_{i, t}^{\max }+\Gamma_{i, t}{ }^{1} \geq 0, \forall i, \forall t=N_{T} \\
1-\sigma_{i, t}^{U} \geq 0, \forall i, \forall t \\
1-\sigma_{i, t}^{D} \geq 0, \forall i, \forall t \\
-\lambda_{j, t, c}^{D}+\lambda_{t}+\nu_{j, t, c}^{\max } \geq 0, \forall j, \forall t, \forall c \\
\mu_{i, t, b}^{\max } \geq 0, \forall i, \forall t, \forall b \\
\nu_{j, t, c}^{\max } \geq 0, \forall j, \forall t, \forall c
\end{gathered}
$$


$\xi_{i, t}^{\min }, \pi_{i, t}^{U}, \pi_{i, t}^{D}, \sigma_{i, t}^{U}, \sigma_{i, t}^{D}, \phi_{i, t}^{U}, \phi_{i, t}^{D}, \chi_{i, t}^{U}, \chi_{i, t}^{D}, \psi_{i, t}^{\max } \geq 0, \forall i, \forall t$.

Constraints (3c)-(3f), (3g)-(3h), (3i), (3j), and (3k) constitute dual constraints with respect to primal variables $g_{i, t, b}, u_{i, t}$, $C_{i, t}^{U}, C_{i, t}^{D}$, and $d_{j, t, c}$, respectively, while constraints (3l)-(3n) express non-negativity of the relevant dual variables.

However, the relaxation of the binary constraints (2) implies that the reformulated LL problem does not generally produce the optimal solution of the original LL problem. In order to ensure that the reformulated LL problem produces a solution that minimally deviates from the solution of the original LL problem, the following primal-dual formulation should be adopted, based on [36]:

$$
\min _{\left\{V^{P}, V^{D}\right\}} \mathrm{DG} \equiv(1 \mathrm{c})-(3 \mathrm{a})
$$

subject to:

$$
(1 \mathrm{e})-(1 \mathrm{t}),(3 \mathrm{c})-(3 \mathrm{n}), \text { and }(1 \mathrm{u}) \text {. }
$$

Problem (4) minimizes the duality gap (DG) between the primal and dual objective function values of the relaxed LL problem (4a), while enforcing the primal (1e)-(1t) and the dual constraints $(3 \mathrm{c})-(3 n)$ of the relaxed LL problem, as well as the original binary constraints $(1 \mathrm{u})$. Although these binary constraints were previously relaxed to enable the derivation of the dual problem, they are enforced in problem (4) to ensure that the solution of this problem conforms to the physical reality of UC.

\section{Final Single-Level Optimization Model}

Formulation (4) constitutes the reformulation of the original LL problem (1c)-(1u). This formulation is similar to the final formulation of [36] which addresses a market clearing and pricing problem from the perspective of the market operator. However, the optimal bidding problem from the perspective of a strategic producer $i$ we aim at solving in this paper is the bi-level problem (1) which additionally contains the UL problem (1a)-(1b). In order to achieve this in a mathematically rigorous fashion, we convert this bi-level problem to a singlelevel problem by:

1) Combining the objective functions of the UL problem (1a) and the reformulated LL problem (4) into a single objective function (5a). In order to do this, we adopt the penalty function method [37] and penalize the DG by a positive constant $W$. By following this approach, the DG is indirectly suppressed (as prescribed by (4a)), while still pursuing a higher producer's $i$ profit (as prescribed by (1a)). The value of the penalty constant $W$ is selected by balancing the trade-off between the accuracy of the market clearing solution and the consideration of the profit. Specifically, small values of $W$ do not sufficiently penalize the DG in (5a), meaning that the proposed model produces a market clearing

\footnotetext{
${ }^{1} \Gamma_{i, t}$ represents the derivative of the contribution of the minimum-up down time constraints (1o)-(1t) to the Lagrangian function of the relaxed LL problem $L^{L L}$ with respect to the variable $u_{i, t}$. Due to the complex form of (1o)-(1t), $\Gamma_{i, t}$ does not adopt a generic expression but a case-specific expression depending on the operating parameters $T_{i}^{U}, T_{i}^{D}, H_{i}^{U}, H_{i}^{D}$ as well as the considered period $t$. These case-specific expressions of $\Gamma_{i, t}$ are provided in Appendix.
}

solution that deviates significantly from the solution of the original market clearing problem, and producer $i$ receives poor quality feedback regarding the impact of its bidding decisions on the market clearing outcome, ultimately making poor quality bidding decisions. On the other hand, large values of $W$ render the profit in (5a) less important, ultimately leading to less profitable bidding decisions. The impact of different values of $W$ on the performance of the model and the selection of a suitable value are quantitatively analyzed in Section III-B.

2) Enforcing both the constraints of the UL problem (1b) and the constraints of the reformulated LL problem (4b), as expressed by $(5 c)$.

The resulting single-level problem is formulated as follows:

$$
\max _{V}(1 \mathrm{a})-W * \mathrm{DG}
$$

where:

$$
V=\left\{k_{i}, V^{P}, V^{D}\right\}
$$

subject to:

$$
(1 \mathrm{~b}),(1 \mathrm{e})-(1 \mathrm{t}),(3 \mathrm{c})-(3 \mathrm{n}) \text {, and }(1 \mathrm{u}) \text {. }
$$

At this point, it should be stressed that this model (5) is merely a tool used by producer $i$ to determine its strategic bidding decision $k_{i}$ and is not generally fully accurate, as the proposed reformulation of the LL problem implies that it does not generally produce the optimal solution of the original LL problem (Section II-C). As a result, the profit (1a) determined by the solution of (5) is the profit estimated by producer $i$ based on the proposed model (we will refer to it as estimated profit in the remainder) and is not generally equal to its actual profit, which is ultimately determined after inputting the value of $k_{i}$ obtained from model (5) to the actual UC clearing algorithm (1c)-(1u).

Going further, it should be noted that problem (5) is nonlinear, since it includes the bilinear terms $\sum_{t, b} \lambda_{t} g_{i, t, b}$ and $\sum_{t, b} k_{i} \lambda_{i, b}^{G} g_{i, t, b}$ in the objective function (5a), and thus any solution obtained by commercial solvers is not guaranteed to be globally optimal. Therefore, we aim at transforming (5) to a mixed-integer linear problem (MILP) which can be efficiently solved to global optimality using commercial branch-and-cut solvers [8]. It should be noted that similar bilinear terms are also present in state-of-the-art MPEC models, where they are handled by adopting linearization techniques exploiting the strong duality theorem [9]. However, such techniques are not applicable to the proposed model, since its LL problem is non-convex and therefore strong duality does not hold.

In order to address this challenge, the binary expansion approach [5], [7] is employed. Let $\left\{g_{i, t, b, l}, l=1,2, \cdots, L\right\}$ be a set of discrete values in the range $\left[0, g_{i, b}^{\max }\right]$ as prescribed by the physical bounds (1f). Then, the variable $g_{i, t, b}$ can be expressed as the following sum of binary variables:

$$
g_{i, t, b}=\sum_{n=0}^{\log _{2}^{L}-1} 2^{n} \triangle_{i, b} x_{i, t, b, n}, \forall i, \forall t, \forall b
$$

where $\triangle_{i, b}=\frac{g_{i, b}^{\max }}{L-1}$ and $x_{i, t, b, n}$ is an auxiliary binary variable. Multiplying both sides of (6a) by $\lambda_{t}$, summing for every $t$ and $b$, and defining a dummy variable $z_{i, t, b, n}$, results in:

$$
\sum_{t, b} \lambda_{t} g_{i, t, b}=\sum_{t, b, n} 2^{n} \triangle_{i, b} z_{i, t, b, n}
$$




$$
z_{i, t, b, n}=\lambda_{t} x_{i, t, b, n}, \forall i, \forall t, \forall b, \forall n
$$

Therefore, the bilinear term $\sum_{t, b} \lambda_{t} g_{i, t, b}$ can be replaced by the expression in the right side of $(6 \mathrm{~b})$ which is linear. The product of variables in (6c) can be transformed into the following equivalent mixed-integer linear constraints:

$$
\begin{gathered}
0 \leq \lambda_{t}-z_{i, t, b, n} \leq M\left(1-x_{i, t, b, n}\right), \forall i, \forall t, \forall b, \forall n \\
0 \leq z_{i, t, b, n} \leq M x_{i, t, b, n}, \forall i, \forall t, \forall b, \forall n
\end{gathered}
$$

where $M$ is a positive constant that is large enough for (6d) and (6e) to hold when $x_{i, t, b, n}=0$ and $x_{i, t, b, n}=1$, respectively. In the first case, (6d) and (6e) are transformed to $0 \leq \lambda_{t} \leq M$ and $z_{i, t, b, n}=0$, respectively. In the second case, (6d) and (6e) are transformed to $\lambda_{t}=z_{i, t, b, n}$ and $0 \leq z_{i, t, b, n} \leq M$, respectively, which again implies $\lambda_{t} \leq M$. Consequently, the value of $M$ should be set according to the upper bound of the market clearing price variable $\lambda_{t}$. In the examined problem, this upper bound is equal to the maximum marginal cost that can be offered by a strategic producer i.e. $\max _{i, b} \lambda_{i, b}^{G} k_{i}$. The other bilinear term $\sum_{t, b} k_{i} \lambda_{i, b}^{G} g_{i, t, b}$ can be linearized in a similar fashion. The set of decision variables of the MILP reformulation of problem (5) includes the set (5b) as well as the aforementioned auxiliary variables introduced for linearizing the two bilinear terms.

The overall structure of the proposed modeling approach, detailed in Sections II-B, II-C and II-D, is illustrated in Fig. 1.

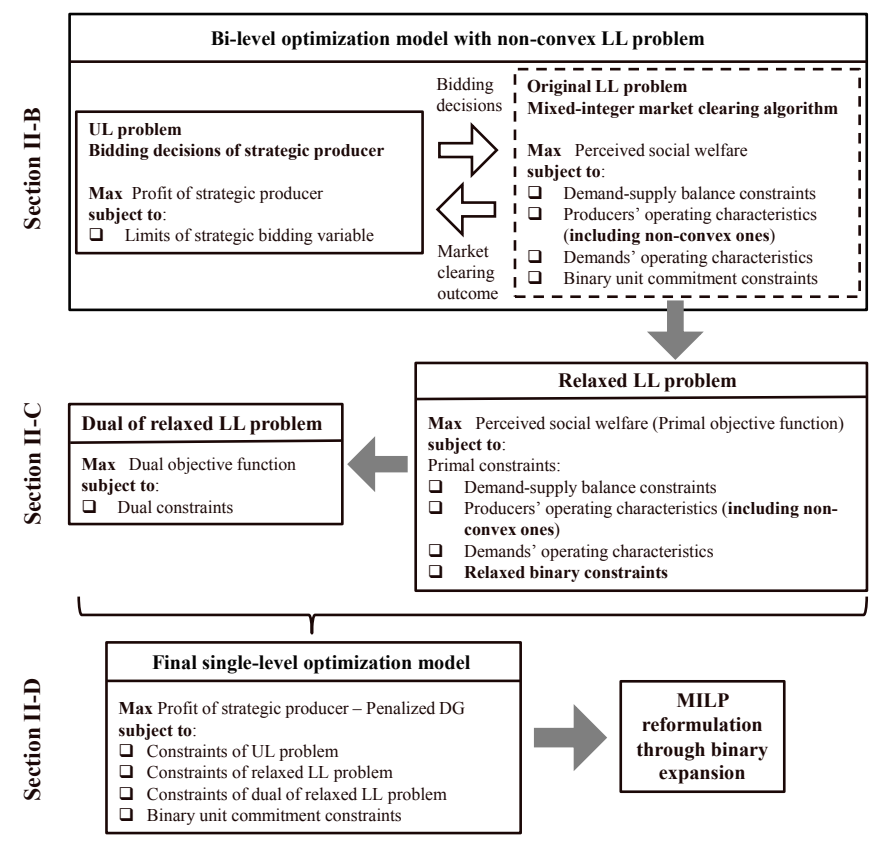

Fig. 1. Illustration of proposed modeling approach.

\section{CASE STUdies}

\section{A. Test Data and Implementation}

Case studies are carried out on the test market of [38], which involves a day-ahead horizon, hourly resolution and 7 electricity producers, the operating parameters of which are presented in Table I. In order to align the data of [38] with the formulation of this paper, the quadratic variable cost curve employed in [38] has been transformed to a piecewise linear curve, by employing the technique presented in [39] and assuming five blocks for each producer. For simplicity and without loss of generality, it is assumed that $H_{i}^{U}=H_{i}^{D}=$ $0, \forall i[39]$.

\begin{tabular}{|c|c|c|c|c|c|c|c|}
\hline Producer $i$ & 1 & 2 & 3 & 4 & 5 & 6 & 7 \\
\hline$f_{i}(\mathfrak{\xi} / \mathbf{h})$ & 18,431 & 17,005 & 13,755 & 9,930 & 9,900 & 8,570 & 7,530 \\
\hline$\lambda_{i, 1}^{G}(\mathfrak{f} / \mathrm{MWh})$ & 6.20 & 32.10 & 36.47 & 64.28 & 84.53 & 97.36 & 102.69 \\
\hline$\lambda_{i, 2}^{G}(\mathfrak{f} / \mathbf{M W h})$ & 7.07 & 34.72 & 38.49 & 72.84 & 93.60 & 105.02 & 111.42 \\
\hline$\lambda_{i, 3}^{G}(\mathfrak{f} / \mathrm{MWh})$ & 7.42 & 35.77 & 39.60 & 81.40 & 102.66 & 115.63 & 123.51 \\
\hline$\lambda_{i, 4}^{G}(\mathfrak{£} / \mathbf{M W h})$ & 7.76 & 36.82 & 40.70 & 89.97 & 111.73 & 126.24 & 135.60 \\
\hline$\lambda_{i, 5}^{G}(\mathfrak{f} / \mathrm{MWh})$ & 8.11 & 37.87 & 41.80 & 98.53 & 120.79 & 136.85 & 147.69 \\
\hline$K_{i}^{U}(\mathfrak{E})$ & $4,000,000$ & 325,000 & 142,500 & 72,000 & 55,000 & 31,000 & 11,200 \\
\hline$K_{i}^{D}(\mathfrak{f})$ & 800,000 & 28,500 & 18,500 & 14,400 & 12,000 & 10,000 & 8,400 \\
\hline$g_{i}^{\min }(\mathrm{MW})$ & 3,292 & 2,880 & 1,512 & 667 & 650 & 288 & 275 \\
\hline$g_{i}^{\max }(\mathrm{MW})$ & 6,584 & 5,760 & 3,781 & 3,335 & 3,252 & 2,880 & 2,748 \\
\hline$R_{i}^{U}(\mathrm{MW} / \mathrm{h})$ & 1,317 & 1,152 & 1,512 & 1,334 & 1,951 & 1,728 & 2,198 \\
\hline$R_{i}^{D}(\mathrm{MW} / \mathrm{h})$ & 1,317 & 1,152 & 1,512 & 1,334 & 1,951 & 1,728 & 2,198 \\
\hline$T_{i}^{U}(\mathbf{h})$ & 24 & 20 & 16 & 10 & 8 & 5 & 4 \\
\hline$T_{i}^{D}(\mathbf{h})$ & 24 & 20 & 16 & 10 & 8 & 5 & 4 \\
\hline$u_{i, 0}$ & 1 & 1 & 1 & 1 & 1 & 0 & 0 \\
\hline$g_{i, 0}(\mathrm{MW})$ & 5,268 & 4,608 & 3,025 & 2,668 & 2,602 & 0 & 0 \\
\hline
\end{tabular}

TABLE I

Electricity producers' operating parameters

As previously discussed, the investigated market problem lies in the optimization of the strategic bidding decisions of a single producer. In the examined case studies, this corresponds to either producer 4 or producer 5 of Table I (two different scenarios) while the rest of the producers are assumed to behave competitively. The upper limits of their strategic bidding variables are assumed $k_{4}^{\max }=k_{5}^{\max }=2$. The penalty constant of the DG has been selected as $W=$ 1000 , for the reasons analyzed in Section III-D. The size of the discretization set employed for the binary expansion of variable $g_{i, t, b}$ has been selected as $L=32$, while the value of the positive constant $M$ is set according to the upper bound of the market clearing price variable $\lambda_{t}$ (Section II-D). In the examined case studies, this quantity is equal to $295.39 £ / \mathrm{MWh}$ (as the largest $\lambda_{i, b}^{G}$ is equal to $147.69 \mathrm{f} / \mathrm{MWh}$ according to Table I and the largest $k_{i}$ is equal to its upper bound $k_{i}^{\max }=$ 2). The proposed MILP model has been implemented using the optimization software FICO $^{\mathrm{TM}}$ Xpress [40] and solved through its solver "Xpress-Optimizer", with the accepted optimality gap set equal to $0.1 \%$.

\section{B. Selection of Penalty Constant $W$}

Before proceeding to the studies demonstrating the effectiveness of the proposed model, we quantitatively analyze an important designing aspect of the model, namely the selection of the penalty constant $W$. As discussed in Section II-D, this selection is based on balancing the trade-off between the accuracy of the market clearing solution and the consideration of the profit. In order to quantitatively demonstrate this tradeoff, we execute the proposed model for different values of $W$ 
and compare its respective solutions in terms of: i) the value of $k_{i}$ determined by the proposed model, ii) the value of DG determined by the proposed model, iii) the minimum value of DG given the value of $k_{i}$ determined by the proposed model; this is obtained by solving problem (4) for this value of $k_{i}$, iv) the estimated profit of producer $i$ determined by the proposed model, and $\mathrm{v}$ ) the actual profit of producer $i$, obtained after inputting the value of $k_{i}$ determined by the proposed model to the actual UC clearing algorithm (1c)-(1u). The results of this analysis are included in Tables II and III, corresponding to two different scenarios where producer 4 and producer 5 optimize their strategic decisions, respectively.

TABLE II

Performance of Proposed Model for Different Values of the Penalty Constant $W$ (Optimizing the Bidding Decisions of Producer 4)

\begin{tabular}{cccccc}
\hline $\boldsymbol{W}$ & $\boldsymbol{k}_{\mathbf{4}}$ & DG (£) & $\begin{array}{c}\text { Minimum } \\
\text { DG (£) }\end{array}$ & $\begin{array}{c}\text { Estimated } \\
\text { profit }(\mathfrak{f})\end{array}$ & $\begin{array}{c}\text { Actual } \\
\text { profit }(\mathfrak{f})\end{array}$ \\
\hline \hline 1 & 1.1836 & 238,587 & 209,676 & $1,819,014$ & $1,477,990$ \\
\hline 10 & 1.2139 & 187,686 & 175,442 & $1,766,701$ & $1,513,320$ \\
\hline 100 & 1.2214 & 153,115 & 144,338 & $1,673,052$ & $1,588,290$ \\
\hline 1,000 & 1.2305 & 129,270 & 129,270 & $1,626,409$ & $1,627,065$ \\
\hline 10,000 & 1.6400 & 87,514 & 87,514 & 630,992 & 630,636 \\
\hline 100,000 & 1.7110 & 73,080 & 73,080 & 435,956 & 436,991 \\
\hline
\end{tabular}

TABLE III

Performance of Proposed Model for Different Values of the Penalty Constant $W$ (Optimizing the Bidding Decisions of Producer 5)

\begin{tabular}{cccccc}
\hline $\boldsymbol{W}$ & $\boldsymbol{k}_{\mathbf{5}}$ & DG (£) & $\begin{array}{c}\text { Minimum } \\
\text { DG (£) }\end{array}$ & $\begin{array}{c}\text { Estimated } \\
\text { profit }(\mathfrak{f})\end{array}$ & $\begin{array}{c}\text { Actual } \\
\text { profit }(\mathfrak{f})\end{array}$ \\
\hline \hline 1 & 1.0877 & 138,976 & 122,135 & 539,353 & 380,443 \\
\hline 10 & 1.2154 & 95,617 & 89,379 & 493.397 & 384,240 \\
\hline 100 & 1.1704 & 86,822 & 81,845 & 469,792 & 405,373 \\
\hline 1,000 & 1.1226 & 74,840 & 74,840 & 451,269 & 455,145 \\
\hline 10,000 & 1.2030 & 42,293 & 42,293 & 419,362 & 427,683 \\
\hline 100,000 & 1.2879 & 28,311 & 28,311 & 335,921 & 334,065 \\
\hline
\end{tabular}

These results demonstrate that relatively small values of $W$ (1-100) do not sufficiently penalize the DG in the objective function (5a) and therefore the obtained DG is higher than the minimum DG corresponding to the obtained $k_{i}$. This means that the model is not as accurate as it could be in approximating the market clearing solution of the original LL problem. In turn, this implies that the strategic producer $i$ employing the proposed model receives poor quality (misleading) feedback regarding the impact of its bidding decisions on the market clearing outcome. As a result, the profit it estimates is far away from the actual profit corresponding to the obtained $k_{i}$; specifically it is much higher than the actual profit, implying that strategic producer $i$ is over-optimistic regarding its profitability in the market.

On the other hand, relatively large values of $W$ (above $1,000)$ rectify the above problems; the obtained DG is equal to the minimum DG corresponding to the obtained $k_{i}$ and the estimated profit is very close to the actual profit corresponding to the obtained $k_{i}$. However, very large values of $W$ (above 10,000) create a different problem. The profit of strategic producer $i$ becomes less important in the objective function (5a) and therefore both the estimated profit and the actual profit are significantly lower.
Ultimately, the most suitable value of $W$ is the one yielding the highest actual profit, which is the ultimate objective of strategic producers employing the proposed model. In the examined case studies, this value is $W=1,000$ (Tables II and III) which is therefore the value employed in the remainder of the quantitative analysis presented in our paper.

\section{Accuracy of Market Clearing Solution}

The aim of the first study lies in validating the accuracy of the proposed model in terms of the obtained market clearing solution. In order to do so, we compare this solution against a benchmark case, where the respective solution is obtained by solving the original mixed-integer clearing algorithm (corresponding to the original LL problem (1c)-(1u)) given the value of $k_{i}$ as determined by the proposed model.

Tables IV and V, corresponding to two different scenarios where producer 4 and producer 5 optimize their strategic bidding decisions, respectively, compare the market clearing solutions in the benchmark case and the proposed model by presenting: i) the dispatch $\left(g_{i, t}=\sum_{b} g_{i, t, b}\right)$ of the 7 producers (and implicitly their UC schedule, since $g_{i, t}>0$ implies $u_{i, t}=1$ while $g_{i, t}=0$ implies $u_{i, t}=0$ ), ii) the prices and iii) the social welfare of the clearing solution. In case the values calculated in the benchmark case and the proposed model are identical, only one entry is given in the respective cell of the Tables. In case they are different, the values calculated in the benchmark case are indicated in parentheses and bold font. The results of Tables IV and V indicate that the market clearing solution calculated by the proposed model is very close to the one calculated by the original mixed-integer clearing algorithm. The UC schedules are identical for all producers and hours in both Tables, while only few and very small deviations are observed in generation dispatch and prices. As a result, the social welfare calculated by the proposed model exhibits a very small deviation with respect to the one calculated in the benchmark case $(0.0002 \%$ and $0.0001 \%$ for the scenario of Table IV and Table V, respectively). These results demonstrate the ability of the proposed model to closely approximate the market clearing solution of the actual mixed-integer clearing algorithm, despite very small deviations, driven by the proposed reformulation of the LL problem (Section II-C) and the employed binary expansion approach (Section II-D). This finding is backed up by the fact that the DG of the proposed model, which implicitly expresses the extent of deviation introduced by the proposed reformulation of the LL problem, is very small: $£ 129,270$ and $£ 74,840$ (or, in relative terms, $0.0928 \%$ and $0.0932 \%$ with respect to the social welfare calculated in the benchmark case), for the scenario of Table IV and Table $\mathrm{V}$, respectively. In other words, the proposed model enables strategic producers to receive very accurate feedback regarding the impact of their bidding decisions on the market clearing outcome.

\section{Added Value with respect to State-of-the-art Models}

The aim of the second study lies in demonstrating the value of the proposed model with respect to state-of-the-art bi-level optimization models, in the context of the investigated market 
TABLE IV

Generation Dispatch (MW), Prices ( $\mathfrak{f} / \mathrm{MWh})$ and Social Welfare $(\mathfrak{f})$ of Market Clearing Solution in Proposed Model and Benchmark Case (given Optimal Bidding Decisions of Producer 4)

\begin{tabular}{|c|c|c|c|c|c|c|c|c|c|c|c|c|c|c|c|c|c|c|c|c|c|c|c|c|}
\hline & \multicolumn{24}{|c|}{ Hour } \\
\hline & 1 & 2 & 3 & 4 & 5 & 6 & 7 & 8 & 9 & 10 & 11 & 12 & 13 & 14 & 15 & 16 & 17 & 18 & 19 & 20 & 21 & 22 & 23 & 24 \\
\hline Producer 1 & 6,584 & 6,584 & 6,584 & 6,584 & 6,584 & 6,584 & 6,584 & 6,584 & 6,584 & 6,584 & 6,584 & 6,584 & 6,584 & 6,584 & 6,584 & 6,584 & 6,584 & 6,584 & 6,584 & 6,584 & $\begin{array}{l}4,584 \\
4\end{array}$ & $4 \quad 6,584$ & 6,5846 & 6,584 \\
\hline Producer 2 & 5,760 & 5,760 & 5,760 & 5,760 & 5,760 & 5,760 & 5,760 & 5,760 & 5,760 & 5,760 & 5,760 & 5,760 & 5,760 & 5,760 & 5,760 & 5,760 & 5,760 & 5,760 & 5,760 & 5,760 & 5,760 & $\begin{array}{ll}0 & 5,760\end{array}$ & 5,7605 & 5,760 \\
\hline Producer 3 & 2,889 & 3,644 & 3,251 & 2,391 & 1,726 & 52,665 & 3,781 & 3,781 & 3,781 & 3,781 & 3,781 & 3,781 & 3,781 & 3,781 & 3,781 & 3,781 & 3,781 & 3,781 & 3,781 & 3,781 & 3,781 & 13,781 & 3,7813 & 3,747 \\
\hline Producer 4 & 1,334 & 667 & 667 & 667 & 667 & 667 & 2,001 & 2,668 & 2,926 & 3,335 & 3,335 & 2,940 & 2,668 & 2,668 & 2,668 & $\begin{array}{c}2,689 \\
(\mathbf{2 , 7 0 2})\end{array}$ & 3,335 & 3,335 & 3,335 & 2,934 & $\begin{array}{l}+2,668 \\
\end{array}$ & $8 \begin{array}{c}2,230 \\
(\mathbf{2 , 2 4 6 )}\end{array}$ & 1,334 & 667 \\
\hline Producer 5 & 650 & 650 & 650 & 650 & 650 & 650 & 1,441 & 2,602 & 2,602 & 2,664 & 2,736 & 2,602 & 2,602 & 2,596 & 2,596 & $\begin{array}{r}2,615 \\
(\mathbf{2 , 6 0 2}) \\
\end{array}$ & 52 & 3,252 & 3,252 & 2,602 & 2,602 & $\begin{array}{r}1,967 \\
(\mathbf{1 , 9 5 1}) \\
\end{array}$ & 859 & 0 \\
\hline Producer 6 & 0 & 0 & 0 & 0 & 0 & 0 & 0 & 997 & 1,584 & 1,584 & 1,584 & 1584 & 1,080 & 936 & 936 & 1584 & 1,710 & 2,232 & 1,584 & 1,584 & $4 \quad 958$ & 0 & 0 & 0 \\
\hline Producer 7 & 0 & 0 & 0 & 0 & 0 & 0 & 0 & 0 & 0 & 0 & 0 & 0 & 0 & 0 & 0 & 0 & 1,511 & 1,701 & 1,428 & 893 & 0 & 0 & 0 & 0 \\
\hline Prices & 40.70 & 41.80 & 41.80 & 39.60 & 38.49 & 40.70 & 102.66 & 115.63 & 120.20 & 120.79 & 120.79 & 120.20 & 115.63 & 111.73 & 111.73 & 120.20 & 126.24 & 4135.60 & 123.51 & 120.20 & 0115.63 & 3110.70 & 93.604 & 41.80 \\
\hline Social welfar & & & & & & & & & & & & $\begin{array}{r}139 \\
(\mathbf{1 3 9}\end{array}$ & $\begin{array}{l}9,311,86 \\
\mathbf{9 , 3 1 2 , 1 8}\end{array}$ & & & & & & & & & & & \\
\hline
\end{tabular}

TABLE V

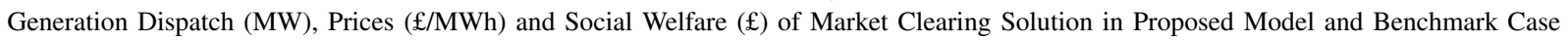
(given Optimal Bidding Decisions of Producer 5)

\begin{tabular}{|c|c|c|c|c|c|c|c|c|c|c|c|c|c|c|c|c|c|c|c|c|c|c|c|c|}
\hline & \multicolumn{24}{|c|}{ Hour } \\
\hline & 1 & 2 & 3 & 4 & 5 & 6 & 7 & 8 & 9 & 10 & 11 & 12 & 13 & 14 & 15 & 16 & 17 & 18 & 19 & 20 & 21 & 22 & 23 & 24 \\
\hline Producer 1 & 6,584 & 6,584 & 6,58 & 46,58 & 46,58 & 6,584 & 6,584 & 6,584 & 6,584 & 6,584 & 6,584 & 6,584 & 6,584 & 6,584 & 6,584 & 6,584 & 6,584 & 6,584 & 6,584 & 6,584 & 6,584 & 6,584 & 6,584 & 6,584 \\
\hline Producer 2 & 5,760 & 5,760 & 5,76 & 05,76 & 05,76 & 5,760 & 5,760 & 5,760 & 5,760 & 5,760 & 5,760 & 5,760 & 5,760 & 5,760 & 5,760 & 5,760 & 5,760 & 5,760 & 5,760 & 5,760 & 5,760 & 5,760 & 5,760 & 5,760 \\
\hline Producer 3 & 2,889 & 3,781 & 3,78 & 13,04 & 22,37 & 3,315 & 3,781 & 3,781 & 3,781 & 3,781 & 3,781 & 3,781 & 3,781 & 3,781 & 3,781 & 3,781 & 3,781 & 3,781 & 3,781 & 3,781 & 3,781 & 3,781 & 3,781 & 3,555 \\
\hline Producer 4 & 1,334 & 1,180 & 788 & 667 & 667 & 667 & 2,001 & 3,335 & 3,335 & 3,335 & 3,335 & 3,335 & 3,335 & 3,335 & 3,335 & 3,335 & 3,335 & 3,335 & 3,335 & 3,335 & 3,335 & $\begin{array}{c}3,323 \\
(\mathbf{3}, \mathbf{3 3 5})\end{array}$ & 2,193 & 859 \\
\hline Producer 5 & 650 & 0 & 0 & 0 & 0 & 0 & 0 & 0 & 0 & 1,915 & 1,915 & 1,915 & 1,301 & 1,301 & 1,301 & $\begin{array}{c}1,707 \\
(\mathbf{1 , 7 2 4}) \\
\end{array}$ & $\begin{array}{c}2,724 \\
(\mathbf{2 , 7 3 1})\end{array}$ & 3,252 & 2,602 & 1,951 & 1,951 & $\begin{array}{c}874 \\
(\mathbf{8 6 2})\end{array}$ & 0 & 0 \\
\hline Producer 6 & 0 & 0 & 0 & 0 & 0 & 0 & 1,441 & 1,584 & 2,232 & 1,404 & 1,476 & 946 & 936 & 936 & 936 & $\begin{array}{c}952 \\
(\mathbf{9 3 6})\end{array}$ & 2,232 & 2,232 & 2,151 & 1,584 & 942 & 0 & 0 & 0 \\
\hline Producer 7 & 0 & 0 & 0 & 0 & 0 & 0 & 0 & 1,348 & 1,545 & 893 & 893 & 893 & 778 & 624 & 628 & 893 & $\begin{array}{c}1,519 \\
(\mathbf{1 , 5 1 1}) \\
\end{array}$ & 1,701 & 1,511 & 1,142 & 0 & 0 & 0 & 0 \\
\hline Prices & 40.7 & 8 & 72.8 & 10 & 39.6 & 1.8 & 115. & 3123 & 135.6 & 115.6 & 115.6 & 115.63 & 111.42 & 11. & 11. & 11525 & 135.60 & 135 & 126 & 123.5 & 115.63 & $\begin{array}{c}98.53 \\
(\mathbf{1 0 5 . 0 7})\end{array}$ & 121.01 & 141.80 \\
\hline Social welfare & & & & & & & & & & & & & $\begin{array}{l}39,927, \\
39,927,\end{array}$ & $\begin{array}{l}595 \\
679)\end{array}$ & & & & & & & & & & \\
\hline
\end{tabular}

problem i.e. the optimization of a strategic producer's bidding decisions. In order to do so, we optimize the strategic decision variables $k_{4}$ and $k_{5}$ of producer 4 and producer 5 (two different scenarios) and calculate the corresponding actual profits they would make in the market, through three different models:

State-of-the-art: This bi-level optimization model does not include the binary UC variables and considers only the variable costs, the maximum output limits and the ramp constraints of the producers. This model is solved after converting it to a single-level MPEC (Section I-A). The obtained values of $k_{4}$ and $k_{5}$ are then inputted to the mixed-integer clearing algorithm (1c)-(1u) to obtain the actual clearing dispatch and prices and subsequently calculate the actual profits of producers 4 and 5 .

Proposed model: This is the model developed in this paper which includes the binary UC variables and therefore considers the non-convex operating characteristics of the producers. The obtained values of $k_{4}$ and $k_{5}$ are inputted to the mixed-integer clearing algorithm (1c)-(1u) to obtain the actual clearing dispatch and prices and subsequently calculate the actual profits of producers 4 and 5 .

Enumeration model: This model adopts a brute-force enumeration approach; it tries out $K$ candidate values of the strategic decision variables $k_{4}$ and $k_{5}$ in the range $\left[1, k_{i}^{\max }\right]$ (with a step of $\left(k_{i}^{\max }-1\right) / K$ ) by inputting them to the market clearing algorithm (1c)-(1u) and calculating the respective profits of producers 4 and 5 . The value yielding the highest profit is selected. If $K$ is large enough to cover sufficiently the entire feasible space $\left[1, k_{i}^{\max }\right]$, the enumeration model determines with high accuracy the optimal solution or "benchmark" solution of the investigated problem, against which the stateof-the-art and proposed models should be compared. In this section, we select $K=10,000$.

The first two rows of Tables VI and VII present the bidding decisions and the resulting profits of producers 4 and 5, respectively, as determined by the three examined models. The bidding decisions determined by the state-ofthe-art model exhibit significant deviations from the optimal ones (determined by the enumeration model) and consequently the strategic producers' profits are significantly lower than 
TABLE VI

Bidding Decisions and Profits of Producer 4

\begin{tabular}{|c|c|c|c|}
\hline & State-of-the-art & Proposed & Enumeration \\
\hline Bidding decision $k_{4}$ & 1.1318 & 1.2305 & 1.2368 \\
\hline $\begin{array}{l}\text { Actual profit without } \\
\text { side payments }(\mathfrak{E})\end{array}$ & $1,484,327$ & $1,627,065$ & $1,662,579$ \\
\hline MWP (E) & 0 & 0 & 0 \\
\hline $\begin{array}{l}\text { Actual profit with } \\
\text { MWP }(£)\end{array}$ & $1,484,327$ & $1,627,065$ & $1,662,579$ \\
\hline $\operatorname{LOCP}(\mathfrak{f})$ & 38,826 & 110,410 & 147,501 \\
\hline $\begin{array}{c}\text { Actual profit with } \\
\text { LOCP (f) }\end{array}$ & $1,523,153$ & $1,737,475$ & $1,810,080$ \\
\hline
\end{tabular}

TABLE VII

Bidding Decisions and Profits of Producer 5

\begin{tabular}{|c|c|c|c|}
\hline & State-of-the-art & Proposed & Enumeration \\
\hline Bidding decision $k_{5}$ & 1.1904 & 1.1226 & 1.1147 \\
\hline $\begin{array}{l}\text { Actual profit without } \\
\text { side payments }(\mathfrak{E})\end{array}$ & 412,241 & 455,145 & 455,668 \\
\hline $\operatorname{MWP}(\mathfrak{f})$ & 0 & 0 & 0 \\
\hline $\begin{array}{l}\text { Actual profit with } \\
\text { MWP (£) }\end{array}$ & 412,241 & 455,145 & 455,668 \\
\hline 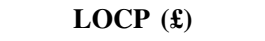 & 226,785 & 255,135 & 646,508 \\
\hline $\begin{array}{l}\text { Actual profit with } \\
\text { LOCP (£) }\end{array}$ & 639,026 & 710,280 & $1,102,176$ \\
\hline
\end{tabular}

the maximum attainable ones $(10.72 \%$ and $9.53 \%$ lower for producer 4 and 5, respectively). On the other hand, the bidding decisions determined by the proposed model are very close to the optimal ones and consequently the strategic producers' profits are significantly higher than the respective profits obtained through the state-of-the-art model (and only $2.14 \%$ and $0.11 \%$ lower than the maximum attainable ones for producer 4 and 5, respectively). These findings demonstrate that the proposed model devises more profitable bidding decisions for strategic producers than state-of-the-art models which neglect the producers' non-convex operating characteristics.

As mentioned in Assumption 1, Section II-A, side payments (uplift payments) are not explicitly considered in the examined modeling framework. The reason lies in the complexity of incorporating (in an already complex model) the side-payments' calculation which takes place separately and after the market clearing process [41]. However, since side payments constitute an important part of markets with complex bidding and UC clearing mechanisms, we have included them in this study through an offline (ex-post) calculation approach. In other words, although the proposed model (as well as the stateof-the-art and the enumeration model) is executed without explicitly considering the side payments to determine the strategic bidding decisions $k_{4}$ and $k_{5}$, the final profits of the two examined producers include the side payments.

In this analysis, we consider the two types of side payments existing in the relevant literature and actual markets [41]: a) make-whole payments (MWP), which ensure that the final profit of each producer is non-negative, i.e. the MWP of each producer is equal to its (potential) profit loss as determined by the market clearing process, and b) lost-opportunity-cost payments (LOCP), which ensure that the final profit of each producer is equal to the maximum profit the producer would make if it was free to individually determine its UC schedule and dispatch according to the market clearing prices (usually referred to as self-scheduling profit), i.e. the LOCP of each producer is equal to the (potential) difference between the selfscheduling profit and the market clearing profit.

The results of this analysis are included in Tables VI and VII. In the examined study, the market clearing profits of the two examined producers are positive (independently of the model employed for determining their strategic bidding decisions) and therefore their MWP are zero and do not alter their final profits. On the other hand, their LOCP are positive and change (increase) their final profits. Despite these changes though, the proposed model still devises more profitable bidding decisions than the state-of-the-art model.

Going further, state-of-the-art bi-level optimization models presented in [2]-[17], [20] investigate the ability of strategic producers to misreport their variable costs (which also applies to the formulation and the previous case studies of this paper), while few of them explore the ability to misreport their maximum generation limits or ramp rates [7], [14], [16]. Since the proposed model enables incorporation of nonconvex operating characteristics, it allows investigating a very interesting aspect that cannot be explored with state-of-theart models: can strategic producers exercise market power and strategically increase their profits by misreporting non-convex cost components or constraints?

Although this issue can potentially involve misreporting different non-convex cost components or constraints, we examine an example case of misreporting the no-load (fixed) cost component. Following the model employed for misreporting the marginal cost component (explained in Assumption 3, Section II-A), the strategic behavior of producer $i$ in terms of its no-load cost is expressed through a decision variable $1 \leq k_{i}^{f} \leq k_{i}^{f, \max }$. If $k_{i}^{f}=1$, producer $i$ behaves competitively and offers its actual no-load cost $f_{i}$ to the market. If $1<k_{i}^{f} \leq k_{i}^{f, \max }$, producer $i$ behaves strategically and offers higher than its actual no-load cost $k_{i}^{f} * f_{i}$ to the market.

The examined example case involves the optimization of the strategic decision variables $k_{4}^{f}$ and $k_{5}^{f}$ of producer 4 and producer 5 (two different scenarios), assuming that they do not misreport their marginal costs (by binding $k_{4}=k_{5}=$ 1 ), and comparing the profits they would make in the market with the corresponding profits in the case they were perfectly competitive $\left(k_{4}^{f}=k_{5}^{f}=1\right)$.

Regarding producer 4 , the results indicate that the optimal value of its strategic variable is $k_{4}^{f}=1$, which implies that this producer cannot strategically increase its profits by misreporting its no-load component. In the case of producer 5 however, the results indicate that the optimal value of its strategic variable is $k_{5}^{f}=1.3645$ and this strategic action of reporting higher than its actual no-load cost in the market increases its profit by $5.24 \%$. Table VIII analyzes the reason behind this result, by comparing the dispatch and different profit components of producer 5 under competitive and strate- 
gic behavior in terms of its no-load cost, during hours 16. Under competitive behavior, producer 5 is committed and produces energy during these hours but the combination of low prices with the incurrence of the no-load cost leads to a significant negative hourly profit. Under strategic behavior, by reporting higher than its actual no-load cost, producer 5 gets out of the merit order and is de-committed at hour 2 (it cannot be de-committed at hour 1 since its initial dispatch is higher than its ramp-down limit, according to Table I); therefore it avoids this negative profit during hours 2-6, apart from the required cost for shutting-down $(£ 12,000$, in line with Table I).

TABLE VIII

Market Outcome for Producer 5 Under Competitive and Strategic Behavior in terms of No-load Cost

\begin{tabular}{|c|c|c|c|c|c|c|c|}
\hline & \multicolumn{6}{|c|}{ Hour } \\
\hline & & 1 & 2 & 3 & 4 & 5 & 6 \\
\hline \multirow{5}{*}{$\begin{array}{c}\text { Competitive } \\
\qquad\left(k_{5}^{f}=1\right)\end{array}$} & Dispatch (MW) & 650 & 650 & 650 & 650 & 650 & 650 \\
\hline & Revenue ( $\mathfrak{f} / \mathbf{h})$ & 26,470 & 27,188 & 27,188 & 25,753 & 25,036 & 26,470 \\
\hline & Var. cost $(\mathfrak{E} / \mathbf{h})$ & 54,980 & 54,980 & 54,980 & 54,980 & 54,980 & 54,980 \\
\hline & Other cost $(\mathfrak{f} / \mathrm{h})$ & 9,900 & 9,900 & 9,900 & 9,900 & 9,900 & 9,900 \\
\hline & Profit $(\mathfrak{f} / \mathrm{h})$ & $-38,410$ & $-37,692$ & $-37,692$ & $-39,127$ & $-39,844$ & $-38,410$ \\
\hline \multirow{5}{*}{$\begin{array}{c}\text { Strategic } \\
\left(k_{5}^{f}=1.3645\right)\end{array}$} & Dispatch (MW) & 650 & 0 & 0 & 0 & 0 & 0 \\
\hline & Revenue (£/h) & 26,470 & 0 & 0 & 0 & 0 & 0 \\
\hline & Var. cost $(\mathfrak{f} / \mathrm{h})$ & 54,980 & 0 & 0 & 0 & 0 & 0 \\
\hline & Other cost $(\mathfrak{f} / \mathrm{h})$ & 9,900 & 12,000 & 0 & 0 & 0 & 0 \\
\hline & Profit $(\mathfrak{k} / \mathbf{h})$ & $-38,410$ & $-12,000$ & 0 & 0 & 0 & 0 \\
\hline
\end{tabular}

These results demonstrate the ability of the proposed model to reveal and quantitatively analyze the potential of strategic behavior in terms of misreporting non-convex operating characteristics, which has not been previously explored and could be very interesting for both strategic producers and market regulators.

\section{E. Analysis of Computational Performance}

The aim of this Section lies in comparing the proposed model against the enumeration model in terms of the tradeoff between accuracy and computational requirements. In this context, we execute the enumeration model for different values of $K$ (number of tested candidate solutions) and compare its respective solutions and computational requirements against the proposed model (Tables IX and X).

As $K$ increases, both the accuracy and the computational requirements of the enumeration model are enhanced. The computational time of the proposed model is in the same order of magnitude with the respective time of the enumeration model with $K=100$, but its resulting strategic producers' profits are significantly higher $(2.94 \%$ and $14.06 \%$ higher for producer 4 and 5, respectively). The enumeration model yields higher profits than the proposed model only in the case with $K$ $=10,000$, but its computational time in this case is significantly higher (more than 50 times higher for both scenarios of Tables IX and X). These results clearly demonstrate that the proposed model achieves a better trade-off between accuracy and computational requirements than the enumeration model.
TABLE IX

Performance of Proposed Model and Enumeration Model (Optimizing the Bidding Decisions of Producer 4)

\begin{tabular}{cccc}
\hline & $\boldsymbol{k}_{\mathbf{4}}$ & Profit (f) & $\begin{array}{c}\text { Computational } \\
\text { time (sec) }\end{array}$ \\
\hline $\begin{array}{c}\text { Enumeration model, } \\
\boldsymbol{K}=10\end{array}$ & 1 & $1,554,520$ & 3 \\
\hline $\begin{array}{c}\text { Enumeration model, } \\
\boldsymbol{K}=100\end{array}$ & 1.24 & $1,580,650$ & 26 \\
\hline $\begin{array}{c}\text { Enumeration model, } \\
\boldsymbol{K}=1,000\end{array}$ & 1.237 & $1,660,020$ & 303 \\
\hline $\begin{array}{c}\text { Enumeration model, } \\
\boldsymbol{K}=10,000\end{array}$ & 1.2368 & $1,662,579$ & 2,732 \\
\hline Proposed model & 1.2305 & $1,627,065$ & 47 \\
\hline
\end{tabular}

TABLE $X$

Performance of Proposed Model and Enumeration Model (Optimizing the Bidding Decisions of Producer 5)

\begin{tabular}{cccc}
\hline & $k_{5}$ & Profit (f) & $\begin{array}{c}\text { Computational } \\
\text { time (sec) }\end{array}$ \\
\hline $\begin{array}{c}\text { Enumeration model, } \\
\boldsymbol{K}=10\end{array}$ & 1.1 & 385,991 & 3 \\
\hline $\begin{array}{c}\text { Enumeration model, } \\
\boldsymbol{K}=100\end{array}$ & 1.12 & 399,046 & 24 \\
\hline $\begin{array}{c}\text { Enumeration model, } \\
\boldsymbol{K}=1,000\end{array}$ & 1.115 & 449,525 & 295 \\
\hline $\begin{array}{c}\text { Enumeration model, } \\
\boldsymbol{K}=10,000\end{array}$ & 1.1147 & 455,668 & 2,506 \\
\hline Proposed model & 1.1226 & 455,145 & 44 \\
\hline
\end{tabular}

\section{Conclusion And Future Work}

Although state-of-the-art bi-level optimization constitutes the most popular methodological framework for developing models of the deregulated electricity market, it neglects the physical non-convex cost components and constraints of market participants, due to its inherent inability to capture binary decision variables in its representation of the market clearing process. This limitation renders it problematic in accurately modeling markets with complex bidding and UC clearing mechanisms which are present in many regions worldwide. This paper has provided a novel modeling approach that enables incorporation of the non-convex generation operating characteristics into the market clearing representation of bilevel optimization market models. This approach is based on the relaxation and primal-dual reformulation of the original, non-convex lower level problem and the penalization of the associated duality gap.

Case studies have demonstrated the effectiveness and value of the proposed approach through three different types of tests. The first one demonstrates that the proposed approach can closely approximate the market clearing solution of the actual UC clearing algorithm, since very small deviations in generation dispatch, prices and social welfare are observed. The second one demonstrates that the strategic producers' bidding decisions devised by the proposed model are more profitable than the respective decisions of the state-of-the-art bi-level optimization approach, and very close to the optimal decisions as calculated through a brute-force enumeration method. In summary, the proposed approach enables strategic 
players to receive more accurate feedback regarding the impact of their bidding decisions on the market clearing outcome and therefore can make better informed bidding decisions, with respect to the state-of-the-art models.

The third test demonstrates the ability of the proposed model to reveal and quantitatively analyze the potential of strategic behavior in terms of misreporting non-convex operating characteristics, which cannot be explored with state-of-theart models. Beyond the example case of the no-load cost analyzed in the presented case studies, similar examples can be conceived for other non-convex characteristics, including startup and shut-down costs, minimum stable generation limits, and minimum-up / down time constraints. A comprehensive analysis of such cases deserves further research efforts.

It should be stressed that the presented model is based on certain assumptions regarding the bidding, clearing and pricing mechanisms of the market (Section I-A). Specifically, the modeled market involves the complex bidding and UC clearing mechanisms adopted in certain markets in the USA and the pricing mechanism proposed in [35], while side payments are not explicitly considered. The integration of the proposed approach in different market designs is a challenging task that deserves further research efforts. Some examples include: a) the bidding and clearing mechanisms of certain European markets (such as CWE and the Nord Pool), where bidding is based on complex orders and clearing is based on an iterative process to deal with paradoxically accepted / rejected orders, b) more complex pricing mechanisms, such as convex hull pricing, which are employed in certain markets in the USA to reduce side payments, and c) side payments which are calculated separately and after the market clearing process. The integration of the proposed approach in such market designs can offer valuable insights regarding the impact of different bidding, clearing and pricing mechanisms on the strategic behavior of market players.

\section{APPENDIX}

This appendix provides the detailed formulation of $\Gamma_{i, t}$, i.e., the derivative of the contribution of the minimum up / down time constraints (1o)-(1t) in the Lagrangian function of the relaxed LL problem $L^{L L}$ with respect to the variable $u_{i, t}$. For simplicity and without loss of generality, it is assumed that $H_{i}^{U}=H_{i}^{D}=0, \forall i$ [39]. The case-specific expressions of $\Gamma_{i, t}$, depending on the minimum up/down times parameters $T_{i}^{U}, T_{i}^{D}$ (assuming $T_{i}^{U}=T_{i}^{D}$ ), and the considered period $t$ are provided as follows:

1) $T_{i}^{U}=T_{i}^{D}=1$ :

$$
\begin{gathered}
\Gamma_{i, t}=\left(\phi_{i, t}^{U}-\phi_{i, t+1}^{U}\right) T_{i}^{U}-\left(\phi_{i, t}^{D}-\phi_{i, t+1}^{D}\right) T_{i}^{D}- \\
\phi_{i, t}^{U}+\phi_{i, t}^{D}, \forall i, \forall t \in\left\{1, \ldots, N_{T}-T_{i}^{U}\right\} ; \\
\Gamma_{i, t}=\phi_{i, t}^{U}(-1)-\phi_{i, t}^{D}\left(T_{i}^{D}-1\right), \forall i, \forall t=N_{T} ;
\end{gathered}
$$

2) $T_{i}^{U}=T_{i}^{D}=2$ :

$$
\begin{gathered}
\Gamma_{i, t}=\left(\phi_{i, t}^{U}-\phi_{i, t+1}^{U}\right) T_{i}^{U}-\left(\phi_{i, t}^{D}-\phi_{i, t+1}^{D}\right) T_{i}^{D}- \\
\sum_{s=1}^{t} \phi_{i, s}^{U}+\sum_{s=1}^{t} \phi_{i, s}^{D}, \forall i, \forall t \in\left\{1, \ldots, T_{i}^{U}\right\} ; \\
\Gamma_{i, t}=\left(\phi_{i, t}^{U}-\phi_{i, t+1}^{U}\right) T_{i}^{U}-\left(\phi_{i, t}^{D}-\phi_{i, t+1}^{D}\right) T_{i}^{D}- \\
\sum_{s=t-T_{i}^{U}+1}^{t} \phi_{i, s}^{U}+\sum_{s=t-T_{i}^{D}+1}^{t} \phi_{i, s}^{D}, \\
\forall i, \forall t \in\left\{T_{i}^{U}+1, \ldots, N_{T}-T_{i}^{U}\right\} ; \\
\Gamma_{i, t}=\phi_{i, t}^{U} T_{i}^{U}-\phi_{i, t}^{D} T_{i}^{D}-\sum_{s=t-T_{i}^{U}+1}^{t} \phi_{i, s}^{U}+ \\
\sum_{s=t-T_{i}^{D+1} \phi_{i, s}^{D}-\left(\chi_{i, t+1}^{U}-\chi_{i, t+1}^{D}\right)\left(N_{T}-t\right),}^{\forall i, \forall t=N_{T}-T_{i}^{U}+1 ;} \\
\sum_{i, t}=-\sum_{s=t-T_{i}^{U}+1}^{N_{T}-T_{i}^{U}+1} \phi_{i, s}^{U}+\sum_{s=t-T_{i}^{D}+1}^{N_{T}-T_{i}^{D}+1} \phi_{i, s}^{D}+ \\
\left(\chi_{i, t}^{U}-\chi_{i, t}^{D}\right)\left(N_{T}-t+1\right)- \\
\chi_{i, s}^{U}+\sum_{s=N_{T}-T_{i}^{D}+2}^{t} \chi_{i, s}^{D}, \forall i, \forall t=N_{T} .
\end{gathered}
$$

3) $T_{i}^{U}=T_{i}^{D}=3, \ldots, 11$ :

$$
\begin{gathered}
\Gamma_{i, t}=\left(\phi_{i, t}^{U}-\phi_{i, t+1}^{U}\right) T_{i}^{U}-\left(\phi_{i, t}^{D}-\phi_{i, t+1}^{D}\right) T_{i}^{D}- \\
\sum_{s=1}^{t} \phi_{i, s}^{U}+\sum_{s=1}^{t} \phi_{i, s}^{D}, \forall i, \forall t \in\left\{1, \ldots, T_{i}^{U}\right\} ; \\
\Gamma_{i, t}=\left(\phi_{i, t}^{U}-\phi_{i, t+1}^{U}\right) T_{i}^{U}-\left(\phi_{i, t}^{D}-\phi_{i, t+1}^{D}\right) T_{i}^{D}- \\
\sum_{s=t-T_{i}^{U}+1}^{t} \phi_{i, s}^{U}+\sum_{s=t-T_{i}^{D+1}}^{t} \phi_{i, s}^{D}, \\
\forall i, \forall t \in\left\{T_{i}^{U}+1, \ldots, N_{T}-T_{i}^{U}\right\} ; \\
\Gamma_{i, t}=\phi_{i, t}^{U} T_{i}^{U}-\phi_{i, t}^{D} T_{i}^{D}-\sum_{s=t-T_{i}^{U}+1}^{t} \phi_{i, s}^{U}+ \\
\sum_{s=t-T_{i}^{D}+1}^{t} \phi_{i, s}^{D}-\left(\chi_{i, t+1}^{U}-\chi_{i, t+1}^{D}\right)\left(N_{T}-t\right), \\
\forall i, \forall t=N_{T}-T_{i}^{U}+1 ; \\
\Gamma_{i, t}=-\sum_{s=t-T_{i}^{U}+1}^{N_{T}-T_{i}^{U}+1} \phi_{i, s}^{U}+\sum_{s=t-T_{i}^{D}+1}^{N_{T}-T_{i}^{D}+1} \phi_{i, s}^{D}+ \\
\left(\chi_{i, t}^{U}-\chi_{i, t}^{D}\right)\left(N_{T}-t+1\right)- \\
\quad\left(\chi_{i, t+1}^{U}-\chi_{i, t+1}^{D}\right)\left(N_{T}-t\right)- \\
\sum_{s=N_{T}-T_{i}^{U}+2}^{t} \chi_{i, s}^{U}+\sum_{s=N_{T}-T_{i}^{D}+2}^{t} \chi_{i, s}^{D}, \\
\forall i, \forall t \in\left\{N_{T}-T_{i}^{U}+2, \ldots, N_{T}-1\right\} ;
\end{gathered}
$$




$$
\begin{gathered}
\Gamma_{i, t}=-\sum_{s=t-T_{i}^{U}+1}^{N_{T}-T_{i}^{U}+1} \phi_{i, s}^{U}+\sum_{s=t-T_{i}^{D}+1}^{N_{T}-T_{i}^{D}+1} \phi_{i, s}^{D}+ \\
\left(\chi_{i, t}^{U}-\chi_{i, t}^{D}\right)\left(N_{T}-t+1\right)-\sum_{s=N_{T}-T_{i}^{U}+2}^{t} \chi_{i, s}^{U}+ \\
\sum_{s=N_{T}-T_{i}^{D}+2}^{t} \chi_{i, s}^{D}, \forall i, \forall t=N_{T} .
\end{gathered}
$$

4) $T_{i}^{U}=T_{i}^{D}=12$ :

$$
\begin{aligned}
& \Gamma_{i, t}=\left(\phi_{i, t}^{U}-\phi_{i, t+1}^{U}\right) T_{i}^{U}-\left(\phi_{i, t}^{D}-\phi_{i, t+1}^{D}\right) T_{i}^{D}- \\
& \sum_{s=1}^{t} \phi_{i, s}^{U}+\sum_{s=1}^{t} \phi_{i, s}^{D}, \forall i, \forall t \in\left\{1, \ldots, T_{i}^{U}\right\} \\
& \Gamma_{i, t}=\phi_{i, t}^{U} T_{i}^{U}-\phi_{i, t}^{D} T_{i}^{D}-\sum_{s=t-T_{i}^{U}+1}^{t} \phi_{i, s}^{U}+ \\
& \sum_{s=t-T_{i}^{D}+1}^{t} \phi_{i, s}^{D},-\left(\chi_{i, t+1}^{U}-\chi_{i, t+1}^{D}\right)\left(N_{T}-t\right), \\
& \forall i, \forall t \in\left\{T_{i}^{U}+1, \ldots, N_{T}-T_{i}^{U}+1\right\} ; \\
& \Gamma_{i, t}=-\sum_{s=t-T_{i}^{U}+1}^{N_{T}-T_{i}^{U}+1} \phi_{i, s}^{U}+\sum_{s=t-T_{i}^{D}+1}^{N_{T}-T_{i}^{D}+1} \phi_{i, s}^{D}+ \\
& \left(\chi_{i, t}^{U}-\chi_{i, t}^{D}\right)\left(N_{T}-t+1\right)- \\
& \left(\chi_{i, t+1}^{U}-\chi_{i, t+1}^{D}\right)\left(N_{T}-t\right)- \\
& \sum_{s=N_{T}-T_{i}^{U}+2}^{t} \chi_{i, s}^{U}+\sum_{s=N_{T}-T_{i}^{D}+2}^{t} \chi_{i, s}^{D}, \\
& \forall i, \forall t \in\left\{N_{T}-T_{i}^{U}+2, \ldots, N_{T}-1\right\} ; \\
& \Gamma_{i, t}=-\sum_{s=t-T_{i}^{U}+1}^{N_{T}-T_{i}^{U}+1} \phi_{i, s}^{U}+\sum_{s=t-T_{i}^{D}+1}^{N_{T}-T_{i}^{D}+1} \phi_{i, s}^{D}+ \\
& \left(\chi_{i, t}^{U}-\chi_{i, t}^{D}\right)\left(N_{T}-t+1\right)-\sum_{s=N_{T}-T_{i}^{U}+2}^{t} \chi_{i, s}^{U}+ \\
& \sum_{s=N_{T}-T_{i}^{D}+2}^{t} \chi_{i, s}^{D}, \forall i, \forall t=N_{T} .
\end{aligned}
$$

5) $T_{i}^{U}=T_{i}^{D}=13, \ldots, 23$ :

$$
\begin{gathered}
\Gamma_{i, t}=\left(\phi_{i, t}^{U}-\phi_{i, t+1}^{U}\right) T_{i}^{U}-\left(\phi_{i, t}^{D}-\phi_{i, t+1}^{D}\right) T_{i}^{D}- \\
\sum_{s=1}^{t} \phi_{i, s}^{U}+\sum_{s=1}^{t} \phi_{i, s}^{D}, \forall i, \forall t \in\left\{1, \ldots, N_{T}-T_{i}^{U}\right\} ; \\
\Gamma_{i, t}=\phi_{i, t}^{U} T_{i}^{U}-\phi_{i, t}^{D} T_{i}^{D}-\sum_{s=1}^{t} \phi_{i, s}^{U}+\sum_{s=1}^{t} \phi_{i, s}^{D}- \\
\left(\chi_{i, t+1}^{U}-\chi_{i, t+1}^{D}\right)\left(N_{T}-t\right), \forall i, \forall t=N_{T}-T_{i}^{U}+1 ;
\end{gathered}
$$

$$
\begin{aligned}
& \Gamma_{i, t}=-\sum_{s=1}^{N_{T}-T_{i}^{U}+1} \phi_{i, s}^{U}+\sum_{s=1}^{N_{T}-T_{i}^{D}+1} \phi_{i, s}^{D}+ \\
& \left(\chi_{i, t}^{U}-\chi_{i, t}^{D}\right)\left(N_{T}-t+1\right)- \\
& \left(\chi_{i, t+1}^{U}-\chi_{i, t+1}^{D}\right)\left(N_{T}-t\right)- \\
& \sum_{s=N_{T}-T_{i}^{U}+2}^{t} \chi_{i, s}^{U}+\sum_{s=N_{T}-T_{i}^{D}+2}^{t} \chi_{i, s}^{D}, \\
& \forall i, \forall t \in\left\{N_{T}-T_{i}^{U}+2, \ldots, T_{i}^{U}\right\} ; \\
& \Gamma_{i, t}=-\sum_{s=t-T_{i}^{U}+1}^{N_{T}-T_{i}^{U}+1} \phi_{i, s}^{U}+\sum_{s=t-T_{i}^{D}+1}^{N_{T}-T_{i}^{D}+1} \phi_{i, s}^{D}+ \\
& \left(\chi_{i, t}^{U}-\chi_{i, t}^{D}\right)\left(N_{T}-t+1\right)- \\
& \left(\chi_{i, t+1}^{U}-\chi_{i, t+1}^{D}\right)\left(N_{T}-t\right)- \\
& \sum_{s=N_{T}-T_{i}^{U}+2}^{t} \chi_{i, s}^{U}+\sum_{s=N_{T}-T_{i}^{D}+2}^{t} \chi_{i, s}^{D}, \\
& \forall i, \forall t \in\left\{T_{i}^{U}+1, \ldots, N_{T}-1\right\} ; \\
& \Gamma_{i, t}=-\sum_{s=t-T_{i}^{U}+1}^{N_{T}-T_{i}^{U}+1} \phi_{i, s}^{U}+\sum_{s=t-T_{i}^{D}+1}^{N_{T}-T_{i}^{D}+1} \phi_{i, s}^{D}+ \\
& \left(\chi_{i, t}^{U}-\chi_{i, t}^{D}\right)\left(N_{T}-t+1\right)- \\
& \sum_{s=N_{T}-T_{i}^{U}+2}^{t} \chi_{i, s}^{U}+\sum_{s=N_{T}-T_{i}^{D}+2}^{t} \chi_{i, s}^{D}, \forall i, \forall t=N_{T} . \\
& \text { 6) } T_{i}^{U}=T_{i}^{D}=24 \text { : } \\
& \begin{array}{c}
\Gamma_{i, t}=\phi_{i, t}^{U} T_{i}^{U}-\phi_{i, t}^{D} T_{i}^{D}-\phi_{i, 1}^{U}+\phi_{i, 1}^{D}- \\
\left(\chi_{i, t+1}^{U}-\chi_{i, t+1}^{D}\right)\left(N_{T}-t\right), \forall i, \forall t=1 ;
\end{array} \\
& \Gamma_{i, t}=-\phi_{i, 1}^{U}+\phi_{i, 1}^{D}+\left(\chi_{i, t}^{U}-\chi_{i, t}^{D}\right)\left(N_{T}-t+1\right)- \\
& \left(\chi_{i, t+1}^{U}-\chi_{i, t+1}^{D}\right)\left(N_{T}-t\right)-\sum_{s=N_{T}-T_{i}^{U}+2}^{t} \chi_{i, s}^{U}+ \\
& \sum_{s=N_{T}-T_{i}^{D}+2}^{t} \chi_{i, s}^{D}, \forall i, \forall t \in\left\{2, \ldots, N_{T}-1\right\} ; \\
& \Gamma_{i, t}=-\phi_{i, 1}^{U}+\phi_{i, 1}^{D}+\left(\chi_{i, t}^{U}-\chi_{i, t}^{D}\right)\left(N_{T}-t+1\right)- \\
& \sum_{s=N_{T}-T_{i}^{U}+2}^{t} \chi_{i, s}^{U}+\sum_{s=N_{T}-T_{i}^{D}+2}^{t} \chi_{i, s}^{D}, \forall i, \forall t=N_{T} .
\end{aligned}
$$

\section{REFERENCES}

[1] D. S. Kirschen and G. Strbac, Fundamentals of power system economics, 2nd ed. West Sussex, U.K.: Wiley, 2018.

[2] B. F. Hobbs, C. B. Metzler, and J.-S. Pang, "Strategic gaming analysis for electric power systems: An mpec approach," IEEE Trans. Power Syst., vol. 15, no. 2, pp. 638-645, May. 2000.

[3] J. D. Weber, T. J. Overbye et al., "An individual welfare maximization algorithm for electricity markets," IEEE Trans. Power Syst., vol. 17, no. 3, pp. 590-596, Aug. 2002.

[4] W. Xian, L. Yuzeng, and Z. Shaohua, "Oligopolistic equilibrium analysis for electricity markets: a nonlinear complementarity approach," IEEE Trans. Power Syst., vol. 19, no. 3, pp. 1348-1355, Aug. 2004. 
[5] M. V. Pereira, S. Granville, M. H. Fampa, R. Dix, and L. A. Barroso, "Strategic bidding under uncertainty: a binary expansion approach," IEEE Trans. Power Syst., vol. 20, no. 1, pp. 180-188, Feb. 2005.

[6] T. Li and M. Shahidehpour, "Strategic bidding of transmissionconstrained gencos with incomplete information," IEEE Trans. Power Syst., vol. 20, no. 1, pp. 437-447, Feb. 2005.

[7] L. A. Barroso, R. D. Carneiro, S. Granville, M. V. Pereira, and M. H. Fampa, "Nash equilibrium in strategic bidding: A binary expansion approach," IEEE Trans. Power Syst., vol. 21, no. 2, pp. 629-638, 2006.

[8] A. G. Bakirtzis, N. P. Ziogos, A. C. Tellidou, and G. A. Bakirtzis, "Electricity producer offering strategies in day-ahead energy market with step-wise offers," IEEE Trans. Power Syst., vol. 22, no. 4, pp. 18041818, Nov. 2007.

[9] C. Ruiz and A. J. Conejo, "Pool strategy of a producer with endogenous formation of locational marginal prices," IEEE Trans. Power Syst., vol. 24, no. 4, pp. 1855-1866, Nov. 2009.

[10] D. Pozo and J. Contreras, "Finding multiple nash equilibria in poolbased markets: A stochastic epec approach," IEEE Trans. Power Syst., vol. 26, no. 3, pp. 1744-1752, Aug. 2011.

[11] C. Ruiz, A. J. Conejo, and Y. Smeers, "Equilibria in an oligopolistic electricity pool with stepwise offer curves," IEEE Trans. Power Syst., vol. 27, no. 2, pp. 752-761, May. 2012.

[12] L. Baringo and A. J. Conejo, "Strategic offering for a wind power producer," IEEE Trans. Power Syst., vol. 28, no. 4, pp. 4645-4654, Nov. 2013.

[13] S. J. Kazempour and H. Zareipour, "Equilibria in an oligopolistic market with wind power production," IEEE Trans. Power Syst., vol. 29, no. 2, pp. 686-697, Mar. 2014.

[14] E. Moiseeva, M. R. Hesamzadeh, and D. R. Biggar, "Exercise of market power on ramp rate in wind-integrated power systems," IEEE Trans. Power Syst., vol. 30, no. 3, pp. 1614-1623, May. 2015.

[15] L. Baringo and A. J. Conejo, "Offering strategy of wind-power producer: A multi-stage risk-constrained approach," IEEE Trans. Power Syst., vol. 31, no. 2, pp. 1420-1429, Mar. 2016.

[16] E. Moiseeva and M. R. Hesamzadeh, "Strategic bidding of a hydropower producer under uncertainty: Modified benders approach," IEEE Trans. Power Syst., vol. 33, no. 1, pp. 861-873, Jan. 2018.

[17] E. Bompard, Y. Ma, R. Napoli, and G. Abrate, "The demand elasticity impacts on the strategic bidding behavior of the electricity producers," IEEE Trans. Power Syst., vol. 22, no. 1, pp. 188-197, Feb. 2007.

[18] S. J. Kazempour, A. J. Conejo, and C. Ruiz, "Strategic bidding for a large consumer," IEEE Trans. Power Syst., vol. 30, no. 2, pp. 848-856, Mar. 2015.

[19] M. G. Vayá and G. Andersson, "Optimal bidding strategy of a plugin electric vehicle aggregator in day-ahead electricity markets under uncertainty," IEEE Trans. Power Syst., vol. 30, no. 5, pp. 2375-2385, Sep. 2015

[20] Y. Ye, D. Papadaskalopoulos, and G. Strbac, "Investigating the ability of demand shifting to mitigate electricity producers' market power," IEEE Trans. Power Syst., vol. 33, no. 4, pp. 3800-3811, Jul. 2018.

[21] A. S. Awad, J. D. Fuller, T. H. El-Fouly, and M. M. Salama, "Impact of energy storage systems on electricity market equilibrium," IEEE Trans. Sustain. Energy, vol. 5, no. 3, pp. 875-885, Jul. 2014.

[22] H. Mohsenian-Rad, "Coordinated price-maker operation of large energy storage units in nodal energy markets," IEEE Trans. Power Syst., vol. 31, no. 1, pp. 786-797, Jan. 2016.

[23] K. Hartwig and I. Kockar, "Impact of strategic behavior and ownership of energy storage on provision of flexibility," IEEE Trans. Sustain. Energy, vol. 7, no. 2, pp. 744-754, Apr. 2016.

[24] Y. Wang, Y. Dvorkin, R. Fernández-Blanco, B. Xu, T. Qiu, and D. S. Kirschen, "Look-ahead bidding strategy for energy storage," IEEE Trans. Sustain. Energy, vol. 8, no. 3, pp. 1106-1117, Jul. 2017.

[25] E. Nasrolahpour, J. Kazempour, H. Zareipour, and W. D. Rosehart, "Impacts of ramping inflexibility of conventional generators on strategic operation of energy storage facilities," IEEE Trans. Smart Grid, vol. 9, no. 2, pp. 1334-1344, Mar. 2018.

[26] S. Boyd and L. Vandenberghe, Convex optimization. Cambridge university press, 2004.

[27] CAISO. (2017) Business practice manual for market instruments. [Online]. Available: https://bpmcm.caiso.com/BPM\%20Document\% 20Library/Market\%20Instruments/BPM_for_Market\%20Instruments_ V44_redline.pdf

[28] PJM. (2018) Manual 11, energy and ancillary services markets operations. [Online]. Available: http://www.pjm.com/ /media/ documents/manuals/m11.ashx
[29] NYISO. (2017) Day ahead scheduling manual. [Online]. Available: $\quad$ http://www.nyiso.com,http://www.nyiso.com/public/ webdocs/markets-operations/documents/Manuals-and-Guides/Manuals/ Operations/dayahd-schd-mnl.pdf

[30] MISO. (2016) Business practices manual energy and operating reserve markets. [Online]. Available: https://www.misoenergy.org/legal/ business-practice-manuals/

[31] P. N. Biskas, D. I. Chatzigiannis, and A. G. Bakirtzis, "European electricity market integration with mixed market designs-part i: Formulation," IEEE Trans. Power Syst., vol. 29, no. 1, pp. 458-465, Jan. 2014.

[32] B. Zeng and L. Zhao, "Solving two-stage robust optimization problems using a column-and-constraint generation method," Oper. Res. Letters, vol. 41, no. 5, pp. 457-461, Sep. 2013.

[33] B. Zeng and Y. An, "Solving bilevel mixed integer program by reformulations and decomposition," Optimization online, pp. 1-34, Jun, 2014.

[34] D. Huppmann and S. Siddiqui, "An exact solution method for binary equilibrium problems with compensation and the power market uplift problem," Eur. J. Oper. Res., vol. 266, no. 2, pp. 622-638, Apr. 2018.

[35] R. P. O’Neill, P. M. Sotkiewicz, B. F. Hobbs, M. H. Rothkopf, and W. R. Stewart Jr, "Efficient market-clearing prices in markets with nonconvexities," Eur. J. Oper. Res., vol. 164, no. 1, pp. 269-285, Jul. 2005.

[36] C. Ruiz, A. J. Conejo, and S. A. Gabriel, "Pricing non-convexities in an electricity pool," IEEE Trans. Power Syst., vol. 27, no. 3, pp. 1334-1342, Aug. 2012.

[37] D. J. White and G. Anandalingam, "A penalty function approach for solving bi-level linear programs," Journal of Global Optimization, vol. 3, no. 4, pp. 397-419, Dec. 1993.

[38] Y. Ye, D. Papadaskalopoulos, and G. Strbac, "Factoring flexible demand non-convexities in electricity markets," IEEE Trans. Power Syst., vol. 30, no. 4, pp. 2090-2099, Jul. 2015.

[39] M. Carrión and J. M. Arroyo, "A computationally efficient mixed-integer linear formulation for the thermal unit commitment problem," IEEE Trans. Power Syst., vol. 21, no. 3, pp. 1371-1378, Aug. 2006.

[40] F. XPRESS. [Online]. Available: http://www.fico.com/en/Products/ DMTools/Pages/FICO-Xpress-Optimization-Suite.aspx

[41] B. Eldridge, R. O'Neill, and B. Hobbs, "Pricing in day-ahead electricity markets with near-optimal unit commitment," Cambridge Working Papers in Economics, Nov. 2018.

\section{BIOGRAPHY}

Yujian Ye (M'12) received the Ph.D. degree from Imperial College London, London, U.K. and is a Research Associate in this institution. His current research focuses on the development and application of game-theoretic as well as agent-based methodologies in energy market modeling.

Dimitrios Papadaskalopoulos (M'13) is a Research Associate at Imperial College London, London, U.K., since 2013. His current research focuses on the development and application of distributed and market-based approaches for the coordination of operation and planning decisions in power systems, employing optimisation and game theoretic principles.

Jalal Kazempour (SM'18) is an Associate Professor in the Department of Electrical Engineering, Technical University of Denmark, Kgs. Lyngby, Denmark. He received his Ph.D. degree in Electrical Engineering from University of Castilla-La Mancha, Ciudad Real, Spain, in 2013. He was a postdoctoral fellow at The Johns Hopkins University, MD, USA, in 2014, and at the Technical University of Denmark in 2015-2016. His research interests include power systems, electricity markets, optimization and its applications to energy systems.

Goran Strbac (M'95) is a Professor of Electrical Energy Systems at Imperial College London, London, U.K. His current research is focused on modelling and optimisation of economic and security performance of energy system operation and investment, including analysis of future energy markets to support cost effective transition to smart low carbon energy future. 